S.D. Ryder, D.J. Pisano, M.A. Walker, and K.C. Freeman, eds.

\title{
Tracing the relation between black holes and dark haloes
}

\author{
Pieter Buyle, Maarten Baes \& Herwig Dejonghe \\ Astronomical Observatory Ghent University, Krijgslaan 281 S9, 9000 \\ Ghent, Belgium
}

\begin{abstract}
We present new velocity dispersion measurements for a set of 12 spiral galaxies and use them to derive a more accurate $v_{c}-\sigma$ relation which holds for a wide morphological range of galaxies. Combined with the $M_{B H}-\sigma$ relation, this relation can be used as a tool to estimate supermassive black hole ( $\mathrm{SMBH})$ masses by means of the asymptotic circular velocity. Together with the Tully-Fisher relation, it serves as a constraint for galaxy formation and evolution models.
\end{abstract}

During the past few years, fiducial mass estimates for the putative SMBHs in the centre of galaxies have become available. These SMBH masses were found to correlate closely to various properties of their host galaxies (Kormendy et al. 1995; Ferrarese et al. 2000; Gebhardt et al. 2000; Graham et al. 2001). Recently, Ferrarese (2002) discovered a tight correlation between the central velocity dispersion and the asymptotic circular velocity measured well into the dark matter dominated region of spiral galaxies. Strikingly, this correlation seems to be satisfied not only by spiral galaxies but also by ellipticals. This indicates a link between the formation and evolution of SMBHs and dark matter haloes.

We took long-slit spectra of 12 galaxies with the EFOSC2 spectrograph on the ESO $3.6 \mathrm{~m}$ telescope. We derived the central velocity dispersions of the galaxies from the $\mathrm{MgI}$ and Fe lines around $520 \mathrm{~nm}$. The asymptotic circular velocities were taken from Palunas \& Williams (2000).

Using linear regression on our data combined with those of Ferrarese (2002) we find the correlation

$$
\log \left(\frac{v_{c}}{u_{0}}\right)=(0.96 \pm 0.11) \log \left(\frac{\sigma}{u_{0}}\right)+(0.21 \pm 0.02) \quad \text { with } u_{0}=200 \mathrm{~km} \mathrm{~s}^{-1}
$$

with a negligible intrinsic scatter of $\chi_{r}^{2}=0.281$ (see figure). We confirm that this relation holds over a large morphological range from late-type spirals to massive ellipticals, and that it appears to break down in the low mass regime $\left(\sigma<80 \mathrm{~km} \mathrm{~s}^{-1}\right)$. Combining the $v_{c}-\sigma$ relation with the equally tight correlation that links the velocity dispersions and $\mathrm{SMBH}$ masses we obtain

$$
\log \left(\frac{M_{B H}}{M_{\odot}}\right)=(4.21 \pm 0.60) \log \left(\frac{v_{c}}{u_{0}}\right)+(7.24 \pm 0.17)
$$



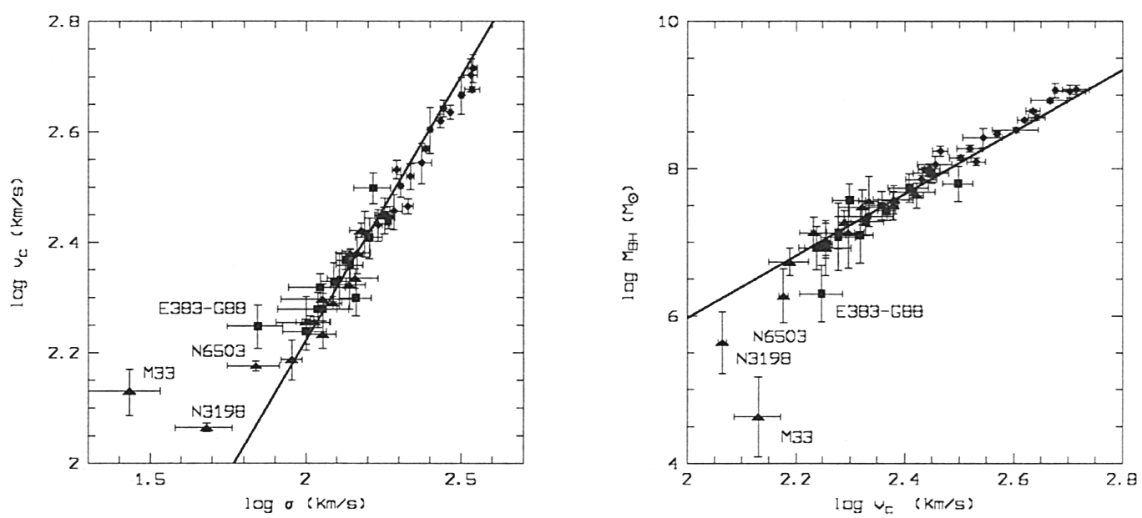

Figure 1. The left and right figure show respectively equations (1) and (2). Ferrarese's data (2002) are represented by triangles, Kronawitter's (2000) by circles and ours by squares. Both relations appear to break down for $\sigma<80 \mathrm{~km} \mathrm{~s}^{-1}$ or $v_{c}<150 \mathrm{~km} \mathrm{~s}^{-1}$.

where the most up-to-date $M_{B H}-\sigma$ relation (Tremaine et al. 2002) was used. This expression can serve as an easy tool to estimate the masses of SMBHs. As the asymptotic circular velocity is a measure for the total gravitational mass, the previous relation can be transformed into a relation between the masses of the SMBH and the dark halo. Considering state-of-the-art high-resolution CDM cosmological simulations (Bullock et al. 2001), we establish a non-linear link

$$
\frac{M_{B H}}{10^{8} M_{\odot}} \sim 0.11\left(\frac{M_{D M}}{10^{12} M_{\odot}}\right)^{1.27}
$$

In conclusion, the $v_{c}-\sigma$ relation together with the $M_{B H}-\sigma$ and TullyFisher relations clearly points towards an intimate interplay between the various components of the galaxies and constitutes a strong test for galaxy formation and evolution models.

\section{References}

Baes, M., Buyle, P., Hau, G. \& Dejonghe, H. 2003, MNRAS, 341, L44

Bullock, J. S. et al. 2001, MNRAS, 321, 559

Ferrarese, L. \& Merritt, D. 2000, ApJ, 539, L9

Ferrarese, L. 2002, ApJ, 578, 90

Gebhardt, K. et al. 2000, ApJ, 539, L13

Graham, A. W., Erwin, P., Trujillo, I. \& Caon, N. 2001, ApJ, 563, L11

Kormendy, J. \& Richstone, D. 1995, ARA\&A, 33, 581

Kronawitter, A. et al. 2000, A\&AS, 144, 53

Palunas, P. \& Williams, T. B. 2000, AJ, 120, 2884

Tremaine, S. et al. 2002, ApJ, 574, 740 